\title{
Synthesis and antiulcer activity evaluation of conjugates of amino acids with $\mathrm{N}$-aroyl- $\mathrm{N}$, $N^{\prime}$-dicyclohexyl urea
}

\author{
Bharat Bhusan Subudhi ${ }^{1 *}$ and Shakti Prasanna Sahoo ${ }^{2}$
}

\begin{abstract}
Amino acids are safe molecules with variable efficacy against ulceration. Considering the good antioxidant potential of $\mathrm{N}$-aroyl- $\mathrm{N}, \mathrm{N}^{\prime}$-dicyclohexyl urea and antiulcer activities of amino acids, a series of amino acid conjugates of $\mathrm{N}$-aroyl- $N, N^{\prime}$-dicyclohexyl urea was synthesized and the effect against ulceration in albino rats induced by pyloric ligation was screened. All these compounds were found to be safe and active. Reduction of ulcer index was significant for all compounds. Conjugates of methionine and histidine exhibited enhanced antiulcer activity comparable to omeprazole in terms of inhibition of release of gastric juice, hydrochloric acid and neutralization activity. The promising efficacy and safety of these compounds is interesting for further investigation.
\end{abstract}

\section{Background}

Intraduodenal administration of mixed amino acid solution has been reported to significantly inhibit gastric acid secretion and gastrin release, stimulated by intragastric perfusion of peptone [1]. However studies conducted with individual amino acids have yielded varying results. Amino acids such as leucine, isoleucine and proline augment the acid release $[2,3]$, where as amino acids including L-serine [4], L-tryptophan, L-arginine, glycine [5,6], tyrosine- and L-glutamic acid [7] have been shown to provide protection against gastric ulcers. Conjugate of methionine with penta-O-acetylglycyrrhizic acid healed both acute and chronic ulcers of rat stomach mucous lining and had low toxicity [8]. Esters of glycine, valine, leucine, proline and tryptophan with labdane diterpene imbricatolic acid exhibited strong gastroprotective action [9]. Although the mechanism of antiulcer action of individual amino acids is not clear, their $\mathrm{N}$-acylated derivatives have been shown to have direct in vitro $\mathrm{H}^{+} / \mathrm{K}^{+}$ATPase inhibitory activity as well as in vivo efficacy in pylorus ligation model [10]. Further radicals are the major causative factor in stress induced gastric ulceration [11] and antioxidants provide effective gastro protection against stress induced ulceration [12].

\footnotetext{
* Correspondence: bharatbhusans@gmail.com

'School of Pharmaceutical Sciences, Siksha O Anusandhan University Bhubaneswar, Orissa, 751003, India

Full list of author information is available at the end of the article
}

With this background and keeping in view the good antioxidant activity of $N$-aroyl- $N, N$-dicyclohexyl urea analogs [13], studies were undertaken to synthesize their amino acid conjugates and evaluate their in vivo antiulcer efficacy against pylorus ligation model.

\section{Results and Discussion Chemistry}

The $N$-cyclohexyl- $N$-(cyclohexylcarbamoyl) benzamide (1) was prepared by reacting $N, N$ '-dicyclohexylcarbodiimide (DCC) with benzoic acid in the reported manner [13]. Equimolar quantity of benzoic acid and DCC were used with triethylamine (TEA) as catalyst. Reaction of dry toluene in presence of TEA generated the carboxylate anion that acted as nucleophile to undergo nucleophillic addition with DCC to give the O-aryl intermediate that upon rearrangement yielded the urea analogue $N$-cyclohexyl- $N$-(cyclohexylcarbamoyl) benzamide (scheme 1). Acylation of $N$-cyclohexyl- $N$-(cyclohexylcarbamoyl) benzamide with chloroacetyl chloride was carried out following reported method $[14,15]$. The TEA acted as catalyst to enhance the nucleophilicity of the secondary amine to attack the nucleophillic centre of the chloroacetyl chloride to give $N$-((2-chloroacetyl) (cyclohexyl) carbamoyl)$N$-cyclohexyl benzamide (scheme 1$)$. The reaction between amino acids and halohydrocarbon has been reported being carried out in presence of $\mathrm{K}_{2} \mathrm{CO}_{3}$ using methanol as solvent [15]. To develop a simple method 
Table 1 The substitution reaction between tyrosine and $\mathrm{N}$-((2-chloroacetyl) (cyclohexyl) carbamoyl) $\mathbf{N}$-cyclohexyl benzamide

\begin{tabular}{ccccc}
\hline No & Solvent & Base & Time (h) & Yield (\%) \\
\hline 1 & $\mathrm{MeOH}$ & $\mathrm{K}_{2} \mathrm{CO}_{3}$ & 9 & 63 \\
2 & $\mathrm{MeOH}$ & $\mathrm{TEA}$ & 8 & 76 \\
3 & $\mathrm{MeOH}$ & $\mathrm{KOH}$ & 8 & 50 \\
4 & $\mathrm{THF}$ & $\mathrm{K}_{2} \mathrm{CO}_{3}$ & 8 & 43 \\
5 & $\mathrm{THF}$ & $\mathrm{TEA}$ & 9 & 48 \\
6 & $\mathrm{THF}$ & $\mathrm{KOH}$ & 10 & 35 \\
7 & Acetonitrile & $\mathrm{K}_{2} \mathrm{CO}$ & 8 & 35 \\
8 & Acetonitrile & $\mathrm{TEA}$ & 8 & 28 \\
9 & Acetonitrile & $\mathrm{KOH}$ & 10 & 22 \\
\hline
\end{tabular}

for substitution reaction of $\mathrm{N}-((2$-chloroacetyl) (cyclohexyl) carbamoyl)- $N$-cyclohexyl benzamide with amino acids, we screened the effect of the base and solvent on the yield of the reaction (Table 1). In this reaction proton is abstracted from the amino group of the amino acids producing the anion that reacts with the nucleophillic centre of the halohydrocarbon. From the table it can be seen that methanol-TEA system effectively promoted this reaction. Other base-solvent systems were less effective in facilitating the reaction. This can be attributed to the good polarity of methanol and appropriate basicity of TEA.

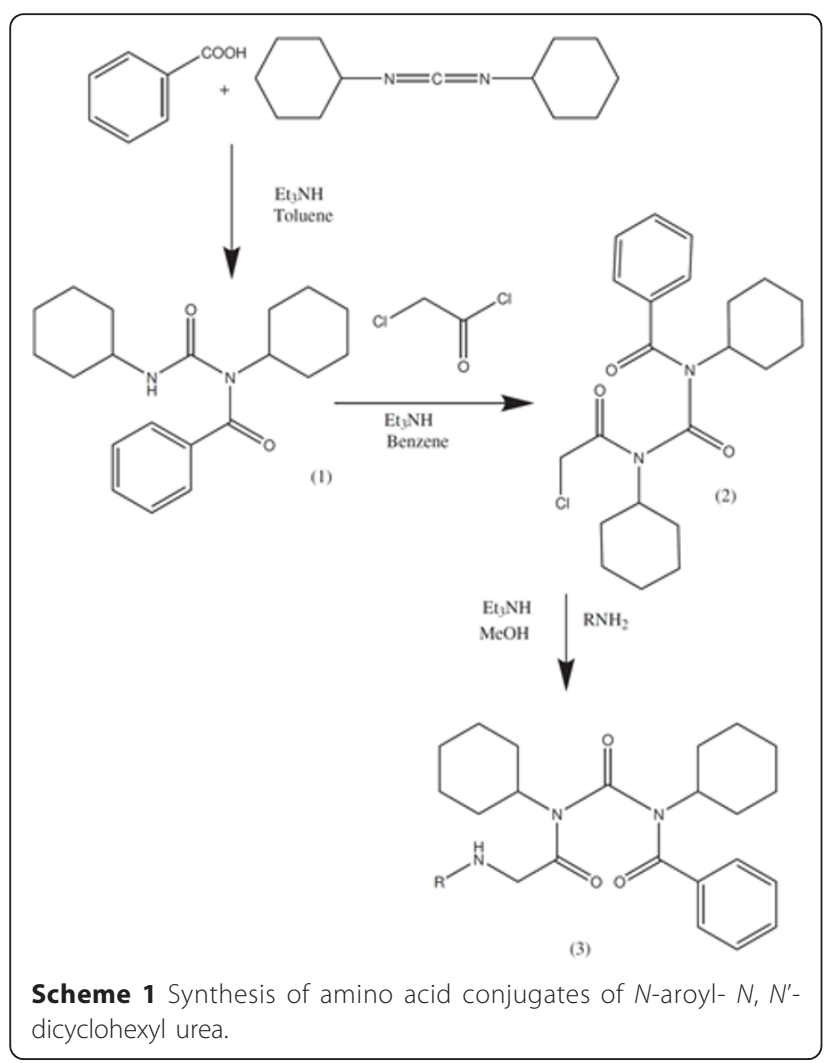

\section{Bioactivity}

Oral acute toxicity study of the synthesized compounds was made by guidelines of Organization of Economic Co-operation and Development (OECD-423) for testing of chemicals. The dose was increased up to $3000 \mathrm{mg} / \mathrm{kg}$ (ip) and lethality was not observed till $3000 \mathrm{mg} / \mathrm{kg}$ indicating the safety of these compounds $(\mathbf{3 a - j})$. The antiulcer effects of the compounds were evaluated on white albino rats $[16,17]$ according to internationally recognized guidelines with approved protocols from institutional animal ethics committee of School of Pharmaceutical Sciences with registration number 1171/ c/08/cpcsea. One day before the induction of ulcers, the animals were divided into groups $(\mathrm{n}=6)$ and drugs/ vehicle was administered to white albino rats. The animals were then fasted (with free access to water) for a period of $24 \mathrm{~h}$, so as to ensure complete gastric emptying and a steady state gastric acid secretion. The $24 \mathrm{~h}$ fasted animals were again administered with the drugs/ vehicle on the morning of the experiment. Sixty minutes after administration of the drug/vehicle, the animals were anaesthetized using anesthetic ether and a midline incision was made just below the xiphoid process. The stomach was lifted out and ligated at the level of the pylorus following which it was replaced and the abdomen wall was closed by interrupted sutures. The animals were kept deprived of food and water and were sacrificed by an overdose of anesthetic ether after $4 \mathrm{~h}$. The stomach was then dissected out, gastric contents were collected and subjected to centrifugation (1000 rpm for $10 \mathrm{~min}$ ) and analysed for volume and $\mathrm{pH}$. Free acidity and total acidity were estimated by titrating $1 \mathrm{~mL}$ of the centrifuged sample diluted to $10 \mathrm{~mL}$ with $0.01 \mathrm{~N} \mathrm{NaOH}$ using Topfer's reagent and phenolphthalein indicator respectively. For estimation of ulcer index inner surface of the stomach was examined for ulceration with magnifying glass. Omeprazole $(1 \mathrm{mg} / \mathrm{kg})$ was used as the standard drug for comparison purpose. The difference between the groups was determined using the one-way analysis of variance (ANOVA).

The newly synthesized compounds exhibited variable antiulcer and antisecretory activity (Table 2). Reduction in volume of gastric juice was significant $(\mathrm{P}<0.001)$ compared to control for $\mathbf{3 a}, \mathbf{3 b}, \mathbf{3 c}, \mathbf{3 d}, \mathbf{3 g}, \mathbf{3 h}, \mathbf{3 i}$ and $3 \mathbf{j}$ indicating their good antisecretory potential. The extent of reduction of secretion of gastric juice for compound $\mathbf{3 b}, \mathbf{3} \mathbf{c}$ and $\mathbf{3 j}$ (Figure 1) was similar to that of omeprazole. The acid neutralizing capacity as indicated by $\mathrm{pH}$ of the gastric fluid was found to be more for compound 3j. All other compounds except 3e, 3f and 3 h exhibited significant $(\mathrm{P}<0.001)$ acid neutralizing capacity. Compound $\mathbf{3 b}$ and $\mathbf{3 c}$ showed antisecretory activity comparable to omeprazole but their acid neutralizing activity was found to be lower than the standard. 
Table 2 Antiulcer activity of compounds (3a-j)

\begin{tabular}{|c|c|c|c|c|c|c|}
\hline Group & $\mathbf{R}$ & Volume(ml) & $\mathrm{pH}$ & Ulcer index & FA (meq./l) & TA (meq./l) \\
\hline Control & - & $6.40 \pm 0.31$ & $1.90 \pm 0.26$ & $16.80 \pm 1.15$ & $44.50 \pm 3.09$ & $48.72 \pm 3.12$ \\
\hline Standard & - & $2.16 \pm 0.28^{\mathrm{a}}$ & $6.10 \pm 0.28^{\mathrm{a}}$ & $2.75 \pm 0.42^{a}$ & $11.48 \pm 1.52^{\mathrm{a}}$ & $12.78 \pm 1.73^{\mathrm{a}}$ \\
\hline $3 a$ & & $4.33 \pm 0.35^{a}$ & $3.50 \pm 0.28^{a}$ & $5.91 \pm 0.51^{a}$ & $33.17 \pm 2.86^{\mathrm{b}}$ & $37.83 \pm 2.28^{b}$ \\
\hline $3 b$ & & $2.83 \pm 0.31^{a}$ & $3.98 \pm 0.25^{\mathrm{a}}$ & $4.45 \pm 0.32^{\mathrm{a}}$ & $22.83 \pm 3.17^{\mathrm{a}}$ & $29.17 \pm 2.66^{a}$ \\
\hline $3 c$ & & $2.38 \pm 0.25^{\mathrm{a}}$ & $4.23 \pm 0.26^{a}$ & $3.81 \pm 0.20^{a}$ & $14.05 \pm 0.97^{a}$ & $16.82 \pm 1.20^{a}$ \\
\hline $3 d$ & & $4.00 \pm 0.36^{a}$ & $4.53 \pm 0.34^{a}$ & $5.01 \pm 0.47^{\mathrm{a}}$ & $27.67 \pm 2.87^{\mathrm{a}}$ & $34.00 \pm 3.36^{a}$ \\
\hline $3 e$ & & $5.03 \pm 0.33$ & $2.61 \pm 0.30$ & $6.93 \pm 0.32^{\mathrm{a}}$ & $26.33 \pm 2.29^{a}$ & $31.67 \pm 2.80^{a}$ \\
\hline $3 f$ & & $4.86 \pm 0.35^{b}$ & $3.06 \pm 0.39$ & $5.85 \pm 0.54^{a}$ & $24.67 \pm 1.99^{a}$ & $29.50 \pm 2.83^{a}$ \\
\hline $3 g$ & & $3.13 \pm 0.44^{\mathrm{a}}$ & $3.51 \pm 0.28^{a}$ & $6.48 \pm 0.41^{\mathrm{a}}$ & $32.17 \pm 2.52 a$ & $38.17 \pm 2.63^{b}$ \\
\hline $3 \mathrm{~h}$ & & $4.38 \pm 0.32^{\mathrm{a}}$ & $3.13 \pm 0.34^{b}$ & $5.31 \pm 0.49^{a}$ & $28.83 \pm 1.81^{\mathrm{a}}$ & $33.33 \pm 1.99^{a}$ \\
\hline $3 \mathbf{i}$ & & $4.03 \pm 0.53^{\mathrm{a}}$ & $3.38 \pm 0.28^{\mathrm{a}}$ & $6.20 \pm 0.40^{a}$ & $30.67 \pm 3.49^{a}$ & $37.17 \pm 3.60^{b}$ \\
\hline \multirow[t]{2}{*}{$3 \mathbf{j}$} & & $2.36 \pm 0.31^{\mathrm{a}}$ & $5.28 \pm 0.11^{\mathrm{a}}$ & $3.31 \pm 0.25$ & $13.32 \pm 1.70^{a}$ & $14.92 \pm 1.86^{\mathrm{a}}$ \\
\hline & & $F=12.95$ & $F=15.58$ & $F=49.34$ & $F=14.72$ & $F=16.89$ \\
\hline
\end{tabular}

Data are represented as mean \pm S.E.M Statistical analysis was done with one way analysis of variance (ANOVA). ${ }^{a} \mathrm{P}<0.001,{ }^{b} \mathrm{P}<0.05$ as compared to control ( $\mathrm{n}$ $=6$ in each group)

Significant $(\mathrm{P}<0.001)$ reduction in free acidity in all compounds except compound $\mathbf{3 a}$ indicated the ability of test compounds to inhibit secretion of hydrochloric acid. This inhibition was higher for the compound $\mathbf{3 j}$ and $\mathbf{3 c}$ followed by that of $\mathbf{3 b}$. Reduction in free acidity of compound $\mathbf{3} \mathbf{j}$ and $\mathbf{3} \mathbf{c}$ was comparable to that of standard. All the compounds that inhibited free acidity, a proportionate inhibitory activity on total acidity was also observed (Figure 2). From the reduction in total acidity value, capacity of these compounds to reduce secretion as well as neutralize acid can be suggested. The inhibition of total acidity was highest for compounds 3c and $3 \mathbf{j}$. The potential of the test compounds for inhibiting hydrochloric acid, gastric juice and neutralizing acidity has resulted in protection of gastric mucosa as observed from the significant $(\mathrm{P}<0.001)$ decrease in ulcer index. From the analysis of the data it was observed that compound $3 \mathbf{c}$ containing methionine as the substituent and $\mathbf{3} \mathbf{j}$ with histidine as the substituent exhibited enhanced antiulcer activity that is comparable to omeprazole in terms of inhibition of release of gastric juice, hydrochloric acid and neutralization activity. 


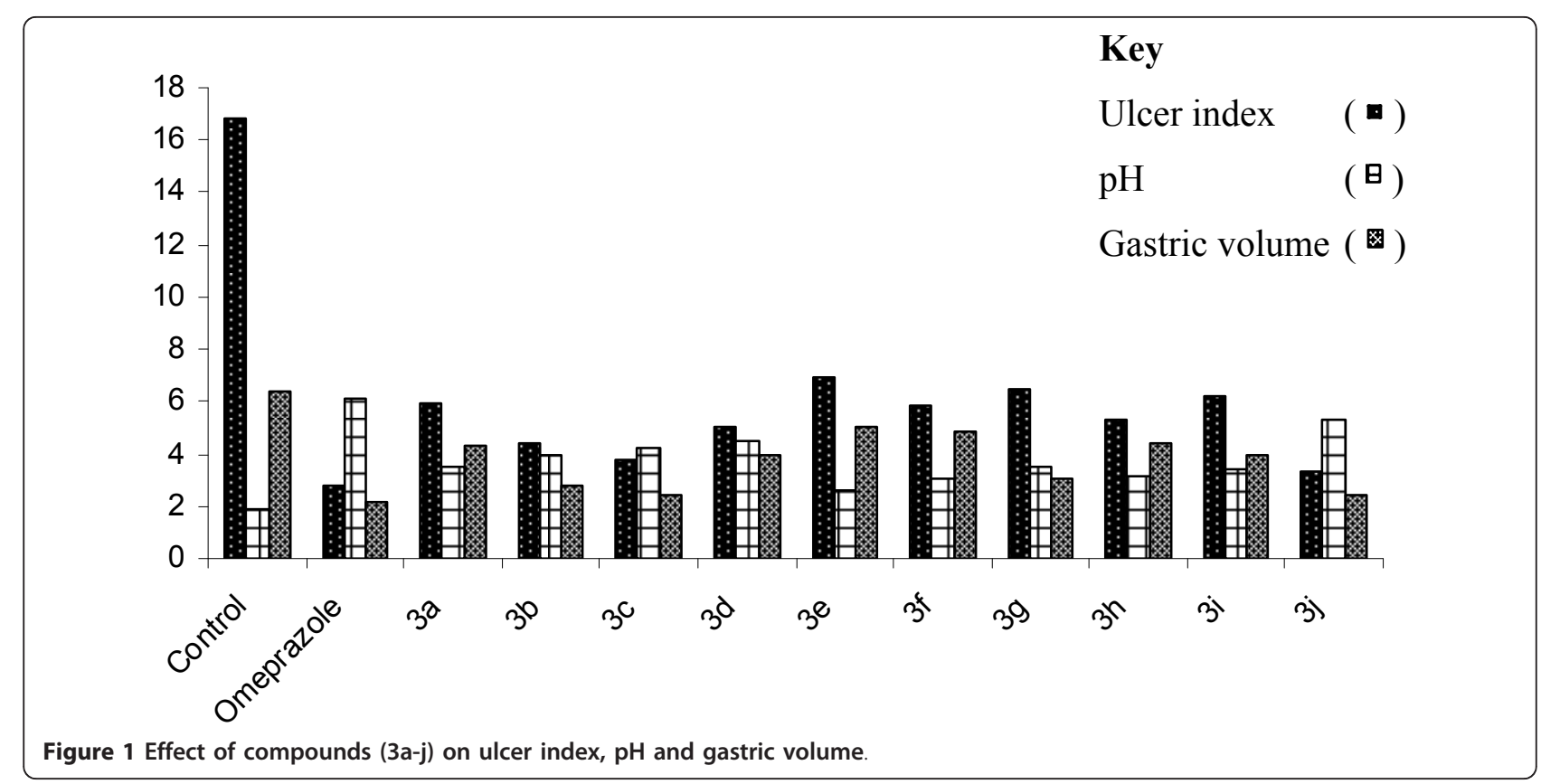

Conjugates of tyrosine, serine, glutamine and cysteine exhibited similar anti-secretory and acid neutralizing capacity. Their antiulcer activity was significant compared to control but were less effective compared to conjugates of methionine and histidine. Conjugates of valine and threonine were least effective in reducing gastric volume. However the reduction of ulcer index was significant. This can be attributed to their effect on reduction of hydrochloric acid as observed from their free acidity and total acidity values. This study has yielded some very safe new compounds with good antiulcer potential.

\section{Experimental}

All reagents and solvents were procured and used without further purification. Melting points were determined

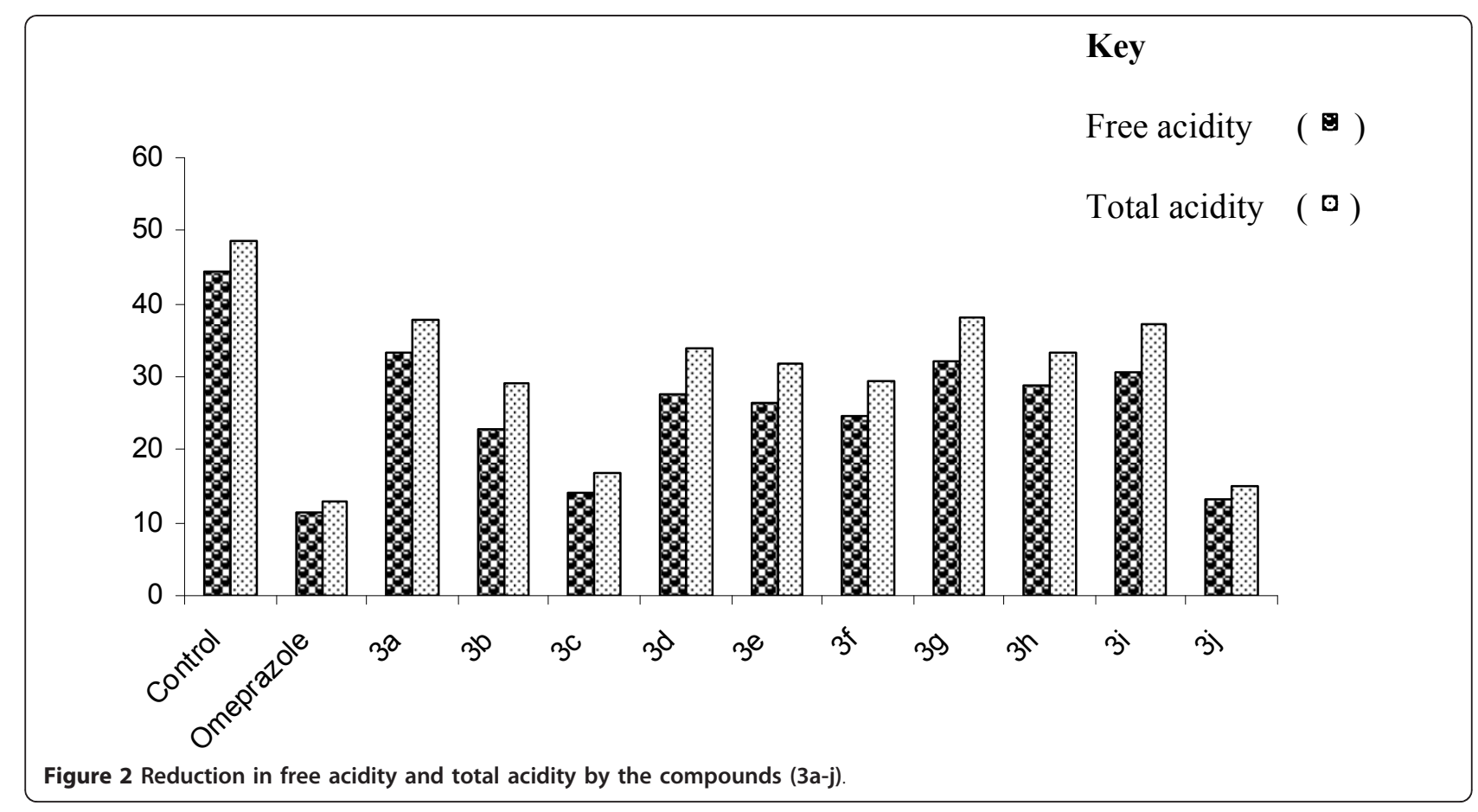


on a Sisco melting point apparatus and are uncorrected. Crude products were purified by column chromatography on silica gel of 60-120 mesh. Molecular weights of the compounds were determined by Rast's procedure. IR spectra were obtained on a JASCO FTIR-4100 spectrometer using $\mathrm{KBr}$ pellet. NMR spectra were recorded on BRUKER AVANCE-II- $400 \mathrm{MHz}$ spectrometer using $\mathrm{CDCl}_{3}$. The chemical shifts were reported as ppm down field using tetramethylsilane (TMS) as an internal standard. Elemental analysis was carried out with PERKIN ELMER-2400 analyzer. Mass spectra were recorded on a MICROMASS Q-TOF MICRO spectrometer operating at $70 \mathrm{eV}$.

\section{$N$-cyclohexyl-N-(cyclohexylcarbamoyl) benzamide (1)}

$N, N$-dicyclohexyl carbodiimide $(1 \mathrm{mmol})$, benzoic acid $(1 \mathrm{mmol})$ and triethylamine $(1 \mathrm{mmol})$ in dry toluene $(50$ ml) was heated up to $110^{\circ} \mathrm{C}$ overnight to yield $N$-benzoyl- $N, N$-dicyclohexylurea (1). The purity of the compound was checked with TLC. Yield 74\%; Off-white; mp 112-115 ${ }^{\circ}$ C; FT-IR $\left(\mathrm{KBr}, \mathrm{cm}^{-1}\right): 3211.46(\mathrm{~N}-\mathrm{H}), 3022.74$ $(\mathrm{Ar}-\mathrm{H}), 2865.12(\mathrm{C}-\mathrm{H}), 1685.12(\mathrm{C}=\mathrm{O}),{ }^{1} \mathrm{H}-\mathrm{NMR}$ $\left(\mathrm{CDCl}_{3}, 400 \mathrm{MHz}\right): 1.12-1.49(20 \mathrm{H}, \mathrm{m}), 5.4(1 \mathrm{H}, \mathrm{s}) 7.6-$ $8.1(5 \mathrm{H}, \mathrm{m})$.

\section{$\mathrm{N}$-((2-chloroacetyl) (cyclohexyl)carbamoyl)N-cyclohexyl benzamide (2)}

To the well stirred solution of $\mathbf{1}(2 \mathrm{mmol})$ and triethylamine $(2.2 \mathrm{mmol})$ in $50 \mathrm{ml}$ benzene, chloro acetyl chloride $(2.2 \mathrm{mmol})$ was added drop by drop for $30 \mathrm{~min}$. It was stirred at room temperature for about 6 h. Progress of the reaction was monitored by TLC. After completion of reaction, the reaction mass was quenched in ice cold water and extracted in diethyl ether. The ether layer was washed twice with $5 \% \mathrm{NaHCO}_{3}$ and twice with distilled water. Finally the ether layer was dried with anhydrous $\mathrm{Na}_{2} \mathrm{SO}_{4}$. Yield $70 \%$; mp 121- $123^{\circ} \mathrm{C}$; White; FT-IR ( $\mathrm{KBr}$, $\left.\mathrm{cm}^{-1}\right)$ : $3034.55(\mathrm{Ar}-\mathrm{H}), 2845.58(\mathrm{C}-\mathrm{H}), 1666.74(\mathrm{C}=\mathrm{O})$; ${ }^{1} \mathrm{H}-\mathrm{NMR}\left(\mathrm{CDCl}_{3}, 400 \mathrm{MHz}\right): 1.11-1.47(20 \mathrm{H}, \mathrm{m}), 3.41$ $(1 \mathrm{H}, \mathrm{m}), 4.17(2 \mathrm{H}, \mathrm{s}), 7.1-8.25(5 \mathrm{H}, \mathrm{m})$.

(2S)-2-((2-3-benzoyl-1, 3-dicyclohexylureido)-2-oxoethyl) amino)- 3- (4-hydroxyphenyl) propanoic acid (3a)

Tyrosine $(1.2 \mathrm{mmol})$ in methanol $(25 \mathrm{ml})$ was neutralized with triethylamine $(1.2 \mathrm{mmol})$. To this $\mathrm{K}_{2} \mathrm{CO}_{3}(600$ $\mathrm{mg}$ ) was added. Later the solution of $2(1 \mathrm{mmol})$ in methanol $(50 \mathrm{ml})$ was added drop by drop for $30 \mathrm{~min}$. The reaction mixture was refluxed for $8 \mathrm{~h}$. The progress of the reaction was monitored by TLC. The reaction mixture was then desolventized and compound was extracted in ethyl acetate. The ethyl acetate layer was washed with water and dried over anhydrous $\mathrm{Na}_{2} \mathrm{SO}_{4}$. The product was further recrystallised from ethanol. Other compounds of the series $\mathbf{3 b} \mathbf{b}-\mathbf{j}$ were obtained in a similar manner. Yield 75\%; mp 124-126 ${ }^{\circ} \mathrm{C}$; Brown; Mol Wt. Anal. Found: 548.85. Calcd. for $\left(\mathrm{C}_{31} \mathrm{H}_{39} \mathrm{~N}_{3} \mathrm{O}_{6}\right)$ : 549.28; FT-IR $\left(\mathrm{KBr}, \mathrm{cm}^{-1}\right): 3442.33(\mathrm{O}-\mathrm{H}), 3029.12(\mathrm{Ar}-$
$\mathrm{H}), 2854.64(\mathrm{C}-\mathrm{H}), 1669.87(\mathrm{C}=\mathrm{O}) ;{ }^{1} \mathrm{H}-\mathrm{NMR}\left(\mathrm{CDCl}_{3}\right.$, $400 \mathrm{MHz}): 1.1-1.49(\mathrm{~m}, 20 \mathrm{H}), 2.12(\mathrm{~s}, 1 \mathrm{H}), 3.45-3.47(\mathrm{~m}$, $1 \mathrm{H}), 3.89(\mathrm{~s}, 1 \mathrm{H}), 5.86(\mathrm{~s}, 1 \mathrm{H}), 6.8-8.1(\mathrm{~m}, 9 \mathrm{H})$. Anal. Found:C, 67.11; H, 8.03; N, 8.01 (\%). Calcd. for $\left(\mathrm{C}_{31} \mathrm{H}_{39} \mathrm{~N}_{3} \mathrm{O}_{6}\right)$ : C, 67.73; H, 7.15; N, 7.64 (\%); MS (m/z): $549\left(\mathrm{M}^{+}\right)$.

(2S)-2-((2-(3-benzoyl-1, 3-dicyclohexylureido)-2-oxoethyl) amino)-3-phenyl propanoic acid (3b)

Yield 64\%; mp 133- $135^{\circ} \mathrm{C}$; White color; Mol Wt. Anal. Found: 532.72. Calcd. for $\left(\mathrm{C}_{31} \mathrm{H}_{39} \mathrm{~N}_{3} \mathrm{O}_{5}\right)$ : 533.29; FT-IR $\left(\mathrm{KBr}, \mathrm{cm}^{-1}\right)$ : $3445.69(\mathrm{O}-\mathrm{H}), 2991.23(\mathrm{Ar}-\mathrm{H}), 2837.85(\mathrm{C}-$ $\mathrm{H}), 1671.42(\mathrm{C}=\mathrm{O}) ;{ }^{1} \mathrm{H}-\mathrm{NMR}\left(\mathrm{CDCl}_{3}, 400 \mathrm{MHz}\right): 1.2-$ $1.45(20 \mathrm{H}, \mathrm{m}), 2.15(1 \mathrm{H}, \mathrm{s}), 3.49-3.51(1 \mathrm{H}, \mathrm{m}), 3.74(1$ H, s), 7.1-8.1 (9 H m); Anal. Found: C, 69.17; H, 8.03; N, 7.25 (\%). Calcd. for $\left(\mathrm{C}_{31} \mathrm{H}_{39} \mathrm{~N}_{3} \mathrm{O}_{5}\right)$ : C, 69.76; $\mathrm{H}, 7.36$; N, $7.87(\%)$;MS $(m / z): 533\left(\mathrm{M}^{+}\right)$.

(2S)-2-((2-(3-benzoyl-1, 3-dicyclohexylureido)-2-oxoethyl) amino)-3-hydroxy butanoic acid (3c)

Yield 72\%; mp 136-138 ${ }^{\circ} \mathrm{C}$; Off white color; Mol Wt. Anal. Found: 487.85. Calcd. for $\left(\mathrm{C}_{26} \mathrm{H}_{37} \mathrm{~N}_{3} \mathrm{O}_{6}\right)$ : 487.27; FT-IR $\left(\mathrm{KBr}, \mathrm{cm}^{-1}\right)$ : $3317.45(\mathrm{O}-\mathrm{H}), 2979.21(\mathrm{Ar}-\mathrm{H})$, 2854.33 (C-H), $1627.03(\mathrm{C}=\mathrm{O})$; ${ }^{1} \mathrm{H}-\mathrm{NMR}\left(\mathrm{CDCl}_{3}, 400\right.$ MHz): 1.05-1.48 $(20 \mathrm{H}, \mathrm{m}), 2.08(1 \mathrm{H}, \mathrm{s}), 3.94-3.99(1 \mathrm{H}$, $\mathrm{m})$, 4-4.016 (1 H, d), 7.26-7.76 (5 H, m); Anal. Found: C, 64.11; $\mathrm{H}, 8.03 ; \mathrm{N}, 8.84$ (\%). Calcd. for $\left(\mathrm{C}_{26} \mathrm{H}_{37} \mathrm{~N}_{3} \mathrm{O}_{6}\right)$ : C, 64.04; H, 7.64; N, 8.61 (\%); $\mathrm{MS}(\mathrm{m} / z): 487\left(\mathrm{M}^{+}\right)$.

(2R)-2-((2-(3-benzoyl-1, 3-dicyclohexylureido)-2-oxoethyl) amino)-3-hydroxy propanoic acid (3d)

Yield 55\%; mp 117-119 ${ }^{\circ} \mathrm{C}$; Pale yellow; Mol Wt. Anal. Found: 473.1. Calcd. for $\left(\mathrm{C}_{25} \mathrm{H}_{35} \mathrm{~N}_{3} \mathrm{O}_{6}\right)$ : 473.55; FT-IR $\left(\mathrm{KBr}, \mathrm{cm}^{-1}\right): 3511.21(\mathrm{O}-\mathrm{H}), 3221.87(\mathrm{~N}-\mathrm{H}), 3011.55(\mathrm{Ar}-$ $\mathrm{H}), 2855.89(\mathrm{C}-\mathrm{H}), 1642.32(\mathrm{C}=\mathrm{O}) ;{ }^{1} \mathrm{H}-\mathrm{NMR}\left(\mathrm{CDCl}_{3}\right.$, $400 \mathrm{MHz}): 1.1-1.74(20 \mathrm{H}, \mathrm{m}), 2(1 \mathrm{H}, \mathrm{s}), 3.64(\mathrm{~s}, 1 \mathrm{H})$, 7.6-8.03 (5 H, m); Anal. Found: C, 63.01; H, 7.11; N, 8.23 (\%). Calcd. for $\left(\mathrm{C}_{25} \mathrm{H}_{35} \mathrm{~N}_{3} \mathrm{O}_{6}\right): \mathrm{C}, 63.40 ; \mathrm{H}, 7.44 ; \mathrm{N}$, $8.87(\%)$.

(2R)-2-((2-(3-benzoyl-1, 3-dicyclohexylureido)-2-oxoethyl) amino)-3-methyl butanoic acid (3e)

Yield 49\%; mp $130-132^{\circ} \mathrm{C}$; Brown; Mol Wt. Anal. Found: 484.79. Calcd. for $\left(\mathrm{C}_{27} \mathrm{H}_{39} \mathrm{~N}_{3} \mathrm{O}_{5}\right)$ : 485.29; FT-IR( $\mathrm{KBr}$, $\left.\mathrm{cm}^{-1}\right)$ : $3487.94(\mathrm{O}-\mathrm{H}), 3189.76(\mathrm{~N}-\mathrm{H}), 3032.65(\mathrm{Ar}-\mathrm{H})$, $2834.95(\mathrm{C}-\mathrm{H}), 1642.78(\mathrm{C}=\mathrm{O}) ;{ }^{1} \mathrm{H}-\mathrm{NMR}\left(\mathrm{CDCl}_{3}, 400\right.$ $\mathrm{MHz})$ : 0.82-0.93 (6 H, m), 1.12-1.44 (20 H, m), 2.34 (1 H, s), 7.31-7.98 (5 H, m); Anal. Found: C, 66.23; H, 7.89; N, 8.12 (\%). Calcd. for $\left(\mathrm{C}_{27} \mathrm{H}_{39} \mathrm{~N}_{3} \mathrm{O}_{5}\right)$ : C, 66.77; $\mathrm{H}, 8.09$; $\mathrm{N}, 8.65(\%)$.

(2R)-2-((2-(3-benzoyl-1, 3-dicyclohexylureido)-2-oxoethyl) amino)-3-(methylthio) propanoic acid (3f)

Yield 56\%; mp 141-143 ${ }^{\circ} \mathrm{C}$; White; Mol Wt. Anal. Found: 502.978. Calcd. for $\left(\mathrm{C}_{27} \mathrm{H}_{39} \mathrm{~N}_{3} \mathrm{O}_{5} \mathrm{~S}\right)$ : 503.25;S, 5.98; FT-IR $\left(\mathrm{KBr}, \mathrm{cm}^{-1}\right)$ : $3456.48(\mathrm{O}-\mathrm{H}), 3211.13(\mathrm{~N}-\mathrm{H}), 3035.21(\mathrm{Ar}-$ $\mathrm{H}), 2865.45(\mathrm{C}-\mathrm{H}), 1654.89(\mathrm{C}=\mathrm{O}) ;{ }^{1} \mathrm{H}-\mathrm{NMR}\left(\mathrm{CDCl}_{3}\right.$, $400 \mathrm{MHz}): 1.11-1.7(20 \mathrm{H}, \mathrm{m}), 2(1 \mathrm{H}, \mathrm{s}), 3.89(1 \mathrm{H}, \mathrm{s})$, 7.6-8.2 (5 H, m); Anal. Found: C, 62.03; H, 7.11; N, 7.67 
(\%). Calcd.for $\left(\mathrm{C}_{27} \mathrm{H}_{39} \mathrm{~N}_{3} \mathrm{O}_{5} \mathrm{~S}\right)$ : C, 62.64; $\mathrm{H}, 7.59 ; \mathrm{N}, 8.11$ (\%); $\mathrm{MS}(\mathrm{m} / \mathrm{z}): 503\left(\mathrm{M}^{+}\right)$.

(2S)-2-((2-(3-benzoyl-1, 3-dicyclohexylureido)-2-oxoethyl) amino)-3-(1H-indol-3-yl) propanoic acid (3g)

Yield 53\%; mp 127-129 $\mathrm{C}$; Off-white; Mol Wt. Anal. Found: 571.65. Calcd. for $\left(\mathrm{C}_{33} \mathrm{H}_{40} \mathrm{~N}_{4} \mathrm{O}_{5}\right)$ : 572.3; O;13.31; FT-IR $\left(\mathrm{KBr}, \mathrm{cm}^{-1}\right): 3445.53(\mathrm{O}-\mathrm{H}), 3240.11(\mathrm{~N}-\mathrm{H})$, 3023.87 (Ar-H), $2854.43(\mathrm{C}-\mathrm{H}), 1651.77(\mathrm{C}=\mathrm{O}) ;{ }^{1} \mathrm{H}-$ NMR $\left(\mathrm{CDCl}_{3}, 400 \mathrm{MHz}\right): 1.2-1.74(20 \mathrm{H}, \mathrm{m}), 2.1(1 \mathrm{H}$, s), 3.54-3.7 (1 H, m), 7.18-8 (9 H, m), 9(1 H, s); Anal. Found: C, 68.68; H, 7.11; N, 9.41 (\%). Calcd. for $\left(\mathrm{C}_{33} \mathrm{H}_{40} \mathrm{~N}_{4} \mathrm{O}_{5}\right):$ C, 69.20; H, 7.03; N, 9, 78 (\%).

5-amino-(2S)-2-((2-(3-benzoyl-1, 3-dicyclohexylureido)-2oxoethyl)amino)-5-oxo pentanoic acid (3h)

Yield 44\%; mp $141-143^{\circ} \mathrm{C}$; White; Mol Wt. Anal. Found: 513.74. Calcd. for $\left(\mathrm{C}_{27} \mathrm{H}_{38} \mathrm{~N}_{4} \mathrm{O}_{6}\right)$ : 514.28; FT-IR ( $\mathrm{KBr}$, $\left.\mathrm{cm}^{-1}\right)$ : $3432.67(\mathrm{O}-\mathrm{H}), 3278.76(\mathrm{~N}-\mathrm{H}), 3176.54(\mathrm{~N}-\mathrm{H})$, 3061.37 (Ar-H), $2845.33(\mathrm{C}-\mathrm{H}), 1648.87$ (C = O); ${ }^{1} \mathrm{H}-$ NMR $\left(\mathrm{CDCl}_{3}, 400 \mathrm{MHz}\right): 1.11-1.74(20 \mathrm{H}, \mathrm{m}), 1.8-1.95$ (4 H, m), 2.1 (1 H, s), 3.49-3.7 (1 H, m), 6.5 (1 H, s), 7.7-8.3 (5 H, m); Anal. Found: C, 62.59; H, 7.11; N, 10.62 (\%). Calcd. for $\left(\mathrm{C}_{27} \mathrm{H}_{38} \mathrm{~N}_{4} \mathrm{O}_{6}\right)$ : C, 63.01; $\mathrm{H}, 7.44 ; \mathrm{N}$, $10.88(\%) ; \mathrm{MS}(\mathrm{m} / z): 514\left(\mathrm{M}^{+}\right)$.

(2R)-2-((2-(3-benzoyl-1, 3-dicyclohexylureido)-2-oxoethyl) amino)-3-mercapto propanoic acid (3i)

Yield 58\%; mp $131-133^{\circ} \mathrm{C}$; Brownish; Mol Wt. Anal. Found: 488.86. Calcd. for $\left(\mathrm{C}_{25} \mathrm{H}_{35} \mathrm{~N}_{3} \mathrm{O}_{5} \mathrm{~S}\right)$ : 489. FT-IR $\left(\mathrm{KBr}, \mathrm{cm}^{-1}\right)$ : $3423.12(\mathrm{O}-\mathrm{H}), 3189.65(\mathrm{~N}-\mathrm{H}), 3033.79$ (Ar$\mathrm{H}), 2843.34(\mathrm{C}-\mathrm{H}), 1666.54(\mathrm{C}=\mathrm{O}) ;{ }^{1} \mathrm{H}-\mathrm{NMR}\left(\mathrm{CDCl}_{3}\right.$, $400 \mathrm{MHz}): 1.1-1.74(20 \mathrm{H}, \mathrm{m}), 2.79-3.1$ (2 H, m), 2 (1 $\mathrm{H}, \mathrm{s})$, 3.52-3.69 (1 H, m), 7.6-8.1 (5 H, m); Anal. Found: C, 54.98; H, 6.32; N, 9.10 (\%). Calcd. for $\left(\mathrm{C}_{25} \mathrm{H}_{35} \mathrm{~N}_{3} \mathrm{O}_{5} \mathrm{~S}\right)$ : C, 61.34; H, 7.15; N, 8.58 (\%).

(2S)-2-((2-(3-benzoyl-1, 3-dicyclohexylureido)-2-oxoethyl) amino)-3-(1-H-imidazole-5-yl) propanoic acid (3j)

Yield 59\%; Brown; Mol Wt. Anal. Found: 522.86. Calcd. for $\left(\mathrm{C}_{28} \mathrm{H}_{37} \mathrm{~N}_{5} \mathrm{O}_{5}\right)$ : 523.28; FT-IR $\left(\mathrm{KBr}, \mathrm{cm}^{-1}\right)$ : $3407.6(\mathrm{O}-$ $\mathrm{H}), 2930.31(\mathrm{Ar}-\mathrm{H}), 2855(\mathrm{C}-\mathrm{H}), 1627.83(\mathrm{C}=\mathrm{O}) ;{ }^{1} \mathrm{H}-$ NMR $\left(\mathrm{CDCl}_{3}, 400 \mathrm{MHz}\right): 1.12-1.88(20 \mathrm{H}, \mathrm{m}), 2.12$ (1 $\mathrm{H}, \mathrm{s}), 2.54-2.56$ (2 H, m), 3.39-3.44 (1 H, t), 3.77-3.81(1 $\mathrm{H}, \mathrm{m}), 7.38-7.85$ (5 H, m), 7.2-8.13 (5 H m,), 8.35 (1 H, s); Anal. Found: C, 64.42; H, 7.48; N, 13.24 (\%). Calcd. for $\left(\mathrm{C}_{28} \mathrm{H}_{37} \mathrm{~N}_{5} \mathrm{O}_{5}\right)$ : C, 64.22; $\mathrm{H}, 7.12 ; \mathrm{N}, 13.37$ (\%); MS $(\mathrm{m} / z): 523.2\left(\mathrm{M}^{+}\right)$.

\section{Acknowledgements}

The authors are thankful to Director, sophisticated analytical instrument facility, Punjab University, Chandigarh for their kind help in analysis.

\section{Author details}

'School of Pharmaceutical Sciences, Siksha O Anusandhan University Bhubaneswar, Orissa, 751003, India. ${ }^{2}$ A.K.R.G. College of Pharmacy, Nallajerla, West Godavari, Andhra Pradesh, 534112, India.

\section{Authors' contributions}

BBS designed the study, carried out the synthesis, characterization, interpretation and drafted the manuscript.

SPS participated in the design of the study, synthesis, characterization and carried out pharmacological and statistical work

\section{Competing interests}

The authors declare that they have no competing interests.

Received: 9 September 2011 Accepted: 20 December 2011 Published: 20 December 2011

\section{References}

1. Ikeda M, Shiratori K, Shimizu K, Watanabe S, Hayashi N: The mechanisms of the inhibition of gastric acid secretion induced by intraduodenal amino acids in rats. Nihon Shokakibyo Gakkai Zasshi 1997, 94:12-20.

2. Feig BW, Nahrwold DL: Leucine and the intestinal phase of gastric acid secretion. Surgery 1988, 104:412-418.

3. Ignatenko LG: Effect of carbon branched-chain amino acids on gastric secretion. Vopr Pitan 1984, 3:51-53.

4. Tariq M, Al Moutaery AR: Gastric anti-ulcer and cytoprotective effect of Iserine in rats. Res Commun Mol Pathol Pharmacol 1997, 97:171-184.

5. Bülbüller N, Akkuş MA, Ilhan YS, Baysal F, Ozercan I, Aygen E, Kirkil C: The effects of L-tryptophan and pentoxiphylline on stress ulcer. Ulus Travma Acil Cerrahi Derg 2003, 9:90-95.

6. Brzozowska I, konturek PC, Brzozowski T, konturek SJ, kwiecien S, Pajdo R, Drozdowicz D, Pawlik M, Ptak A, Hahn EG: Role of prostaglandins, nitric oxide, sensory nerves and gastrin in acceleration of ulcer healing by melatonin and its precursor, L-tryptophan. J Pineal Res 2002, 32:149-162.

7. Yang EY, Nahrwold DL: Tyrosine inhibits intestinal-phase gastric-acid secretion. Surgery 1993, 114:915-920.

8. Kondratenko RM, Baltina LA, Gromakova LS, Khalilov LM, Nasyrov KM, Tolstikov GA: Synthesis and anti-inflammatory activity of 8-substituted 7[thietanyl-3]theophyllines. Pharmaceutical Chemistry Journal 2007, 41:357-361.

9. Hirschmann GS, Pertino MW, Rodriguez JA, Monsalve F, Droguett D, Theoduloz C: Synthesis, gastroprotective effect and cytotoxicity of new amino acid diterpene monoamides and diamides. Molecules 2010, 15:7378-7394.

10. Sharma P, Singh S, Siddiqui TI, Singh VS, Kundu B, Prathipati P, Saxena AK, Dikshit DK, Rastogi L, Dixit C, Gupta MB, Patnaik GK, Dikshit M: alphaAmino acid derivatives as proton pump inhibitors and potent anti-ulcer agents. Eur J Med Chem 2007, 42:386-393.

11. Das D, Bandyopadhyay D, Bhattacharjee M, Banerjee RK: Hydroxyl radical is the major causative factor in stress-induced gastric ulceration. Free Red Biol Med 1997, 23:8-18

12. Potrich FB, Allemand A, da Silva LM, dos Santos AC, Baggio CH, Freitas CS, Mendes DAGB, Andre E, Werner MFDP, Marquesa MCA: Involvement of the antioxidant system: Antiulcerogenic activity of hydroalcoholic extract of Achillea millefolium L. Journal of Ethnopharmacology 2010, 130:85-92.

13. Amornraksa K, worachartcheewan A, Prachayasittikul V: Facile Synthesis of $\mathrm{N}$-Aroylurea Analogs as Potential Antioxidants. European Journal of Scientific Research 2009, 31:510-518.

14. Rigo B, Lespagnol C, Pauly M: Synthesis of N-Acylpyroglutamic Acids Having Bactericide and Fungicide Properties. J Het Chem 1988, 25:59-63.

15. Vijaykumar H, Gnanendra CR, Naik N: Synthesis of amino acid analogues of $5 \mathrm{H}$-dibenz $[\mathrm{b}$, f]azepine and evaluation of their radical scavenging activity. E-Journal of Chemistry 2009, 6:125-132

16. Shay H, Komarov SA, Fels SS, Meranze D, Greenstein M, Siplet H: The Effect of Stress on the Gastric Mucosal Barrier in Rats. Gastroenterology 1945, 5:43-63.

17. Nair V, Arjuman A, Gopalakrishna HN, Dorababu P, Mirshad PV, Bhargavan D, Chatterji D: Evaluation of the anti-ulcer activity of NR-ANXC (a polyherbal formulation) in aspirin \& pyloric ligature induced gastric ulcers in albino rats. Indian J Med Res 2010, 132:218-223.

doi:10.1186/1752-153X-5-86

Cite this article as: Subudhi and Sahoo: Synthesis and antiulcer activity evaluation of conjugates of amino acids with $N$-aroyl- $N, N^{\prime}$-dicyclohexy urea. Chemistry Central Journal 2011 5:86. 